\title{
Pico satélite educativo CanSat desarrollado en las plataformas ARM-CortexM4 y FPGA
}

\author{
Educational pico-satellite CanSat developed on an ARM-CortexM4 and FPGA \\ platforms
}

SÁNCHEZ-TEXIS, Fernando †*, IBARRA-BONILLA, Mariana Natalia y REYES-CASTILLO, Ivan

Instituto Tecnológico Superior de Atlixco, División de Ingeniería Mecatrónica

ID $1^{\mathrm{er}}$ Autor: Fernando, Sánchez-Texis / ORC ID: 0000-0002-1792-8855, CVU CONACYT ID: 95289

ID $1^{\text {er }}$ Coautor: Mariana Natalia, Ibarra-Bonilla / ORC ID: 0000-0001-7123-9105, CVU CONACYT ID: 237756

ID $2^{\text {do }}$ Coautor: Ivan, Reyes-Castillo / ORC ID: 0000-0003-3160-5611, CVU CONACYT ID: 294850

DOI: $10.35429 /$ JEA.2019.20.6.9.17

Recibido: 19 de Junio 2019; Aceptado 30 Septiembre, 2019

\begin{abstract}
Resumen
Este artículo presenta el diseño e implementación de un pico-satélite CanSat, desarrollado en las plataformas ARM-CortexM4 y FPGA Spartan6. La estructura del CanSat consiste de cuatro módulos distribuidos en cuatro niveles de construcción física. El primer nivel es el módulo de sensores, conformado por: el circuito integrado BME280 (barómetro, humedad y temperatura), el sistema de GPS L70 y un sistema de sensores inerciales MPU6050 y AK8975. El segundo nivel es el módulo de control de misión e incorpora un microcontrolador ARM-STM32F407 integrado con el OS-Micropython que permite la programación en lenguaje Python. Este módulo tiene la capacidad de almacenamiento de datos mediante una memoria EEPROM-M24C32 y una micro-SD. El tercer nivel es el módulo de comunicaciones inalámbricas de tecnología LoRa con un alcance de $10 \mathrm{Km}$. El cuarto nivel es el módulo de visión, constituido por una cámara OV7670 interconectada con la FPGA XC6SLX16, que funciona como un sistema de captura de fotos y video. El diseño de los módulos fue realizado con tecnología SMD en PCB de hasta cuatro capas. Se presenta el diseño de la carcasa protectora del CanSat en material PLA, fabricada mediante impresión 3D de tecnología FDM. Se presentan los resultados de pruebas preliminares de funcionamiento.
\end{abstract}

Pico-Satélite, ARM, FPGA

\begin{abstract}
This paper presents the design and implementation of a CanSat pico-satellite developed on the ARM-CortexM4 and FPGA Spartan6 platforms. The CanSat structure consists of four modules distributed in four levels of construction. The first level is the sensor module, consisting of: the IC BME280 (barometer, humidity and temperature), the L70 GPS system and an inertial sensors system MPU6050 and AK8975. The second level is the mission control module and incorporates an ARM-STM32F407 microcontroller integrated with the OS-Micropython that allows programming in Python language. This module has the capacity of data storage using an EEPROM-M24C32 memory and a micro-SD. The third level is the LoRa technology wireless communications module with a $10 \mathrm{Km}$ range. The fourth level is the vision module, consisting of an OV7670 camera interconnected with the FPGA XC6SLX16, which functions as a photo and video capture system. The design of the modules was made with SMD technology in PCB of up to four layers. The design of the CanSat protective case in PLA material, manufactured by 3D printing of FDM technology, is presented. Finally, the results of preliminary performance tests are presented.
\end{abstract}

Pico-Satellite, ARM, FPGA

Citación: SÁNCHEZ-TEXIS, Fernando, IBARRA-BONILLA, Mariana Natalia y REYES-CASTILLO, Ivan. Pico satélite educativo CanSat desarrollado en las plataformas ARM-CortexM4 y FPGA. Revista de Aplicaciones de la Ingeniería. 2019. 6-20: 9-17

\footnotetext{
* Correspondencia del Autor (Correo electrónico: fernando.sanchez@itsatlixco.edu.mx)

$\dagger$ Investigador contribuyendo como primer autor.
} 


\section{Introducción}

La difusión y el conocimiento de los pico y nanosatélites se ha expandido en las últimas décadas, debido en gran parte a la actividad dentro de la comunidad de satélites universitarios. En particular, el desarrollo de pico-satélites CanSat ha abierto una nueva rama en los programas educativos, porque proporcionan la base y un fondo de motivación para que los estudiantes obtengan experiencia práctica en proyectos de tecnología espacial (Bouwmeester \& Guo, 2010). El término CanSat fue introducido en 1999 por el profesor Robert Twiggs del Laboratorio de Desarrollo Espacial de la Universidad de Stanford, quien estableció que la electrónica y los componentes de este dispositivo se pueden organizar dentro de una lata de refresco (Twiggs, 1998).

Los CanSat proporcionan el conocimiento de los principios operativos básicos de un pequeño satélite (Colin \& Jimenez-Lizarraga, 2015), sin embargo no orbitan. Estos son lanzados por un cohete, globo o cualquier vehículo aéreo no tripulado, desde una altura aproximada de un kilómetro, para descender lentamente en paracaídas mientras recopilan y transmiten datos por telemetría a una estación terrestre. Los CanSat son completamente autónomos, pues durante el ascenso, descenso y/o retorno no pueden recibir instrucciones desde la estación terrestre.

Para lograr su misión, un CanSat se puede diseñar con componentes comerciales. Es común que la unidad de control se implemente con un microcontrolador (MCU) comercial y la estación terrestre sea una computadora portátil con una antena conectada. En la literatura se reportan diversos trabajos que utilizan un microcontrolador PIC (Carrasco y Vázquez, 2014) o alguna de las múltiples plataformas embebidas de Arduino (Chaudhry y Mishra, 2015; Paudel et al., 2017; Waleligne, 2018) como unidad principal de control de los componentes del CanSat. Estas dos tecnologías son de fácil manejo y programación, pues en línea existen múltiples proyectos para estudiantes, ingenieros y aficionados. Hoy en día el mercado de la electrónica móvil e integrada está siendo dominado por los microprocesadores y microcontroladores ARM.
La arquitectura ARM es el conjunto de instrucciones de 32 bits más ampliamente utilizado en unidades producidas, y la simplicidad de los procesadores los hace ideales para aplicaciones de baja potencia. A diferencia de los PIC y de las plataformas de Arduino, los microcontroladores ARM permiten ejecutar sistemas operativos robustos. Adicionalmente, los FPGA son arreglos de compuertas programables superiores a los microcontroladores, en cuanto a la capacidad de procesamiento y de reconfiguración de hardware.

Por lo anterior, el principal objetivo de este trabajo es presentar el diseño e implementación del sistema de control de un pico-satélite CanSat, desarrollado en las plataformas ARM-CortexM4 y un FPGA Spartan 6 para cumplir la misión de telemetría. Los MCU ARM-CortexM4 integran una unidad de punto flotante por lo que permite realizar operaciones complejas con mayor precisión, y al ser tecnología de 32 bits permiten el procesamiento de datos de manera más eficiente, además de que son diseñados para un bajo consumo de energía, lo que permite aumentar la vida útil de la batería.

Se propone el uso del FPGA para el control de una cámara de video, pues debido a la capacidad de procesamiento en paralelo que poseen los FPGA son ideales para procesar una gran cantidad de datos a alta velocidad. Esta propuesta contribuye al desarrollo de sistemas de instrumentación y control en sistemas embebidos digitales.

La organización de este artículo es la siguiente: Sección 2 describe el diseño de las estructuras que componen el CanSat. La sección 3 presenta la descripción de los resultados preliminares. Las conclusiones y el trabajo en progreso se presentan en la sección 4.

\section{Descripción general del diseño del CanSat}

El CanSat se diseña para cumplir una misión específica, para este caso es enviar datos de telemetría a una altura de $900 \mathrm{~m}$ y recibirlos en tiempo real en la estación terrestre. Los datos a transmitir son: temperatura interna y externa del CanSat, humedad relativa, altitud, longitud y latitud, nivel de batería, aceleración y fotografía y/o video. 
Todos los componentes y sensores deben caber dentro de una lata de refresco de $355 \mathrm{ml}$. Para cumplir con la misión, se propone incorporar diferentes subsistemas al CanSat, siendo la unidad de control principal un microcontrolador ARM Cortex-M4. Un esquema de los subsistemas que incorpora el CanSat se presenta en la Figura 1. Todos los subsistemas son distribuidos en cuatro niveles de construcción física, tal como presenta el modelo $3 \mathrm{D}$ de la Figura 2.

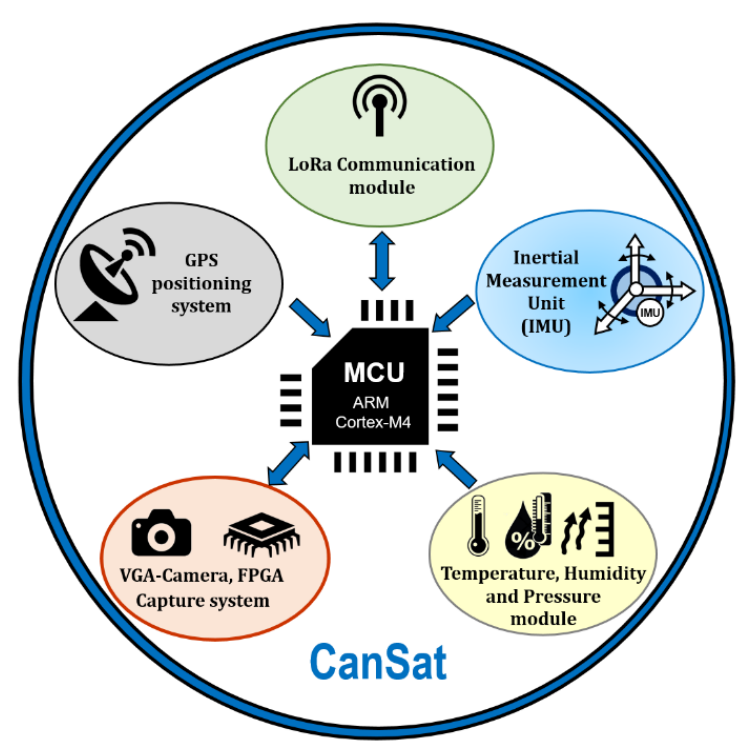

Figura 1 Subsistemas que incorpora el CanSat Fuente: Elaboración Propia

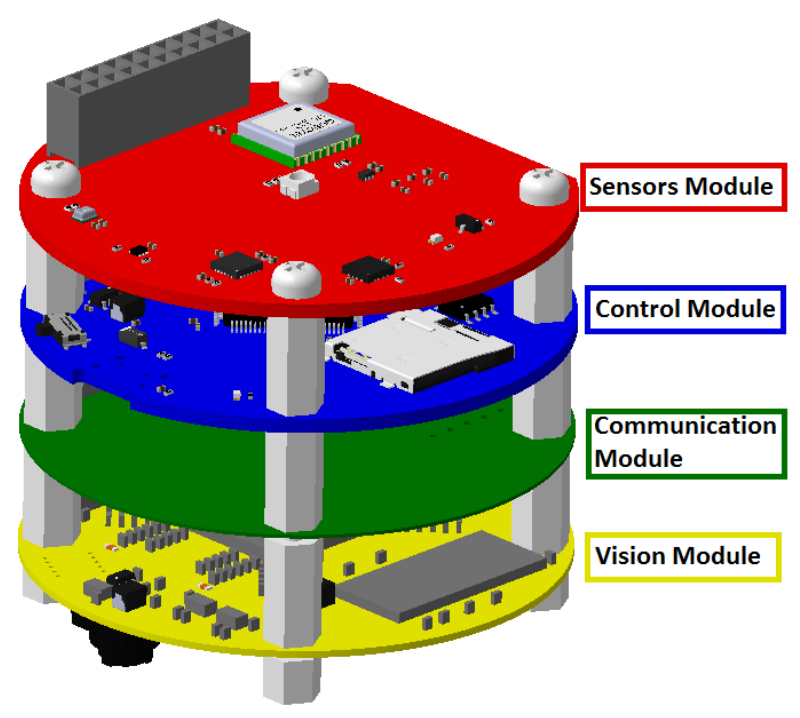

Figura 2 Modelo 3D de la construcción física del CanSat Fuente: Elaboración Propia

\section{Módulo de control}

El módulo de control está constituido por un microcontrolador ARM del fabricante STMicroelectronics, modelo STM32F407. Alrededor del microcontrolador se interconecta una memoria de $4 \mathrm{~Kb}$ M24C32-EEPROM, para el respaldo de datos.
También integra un socket para la inserción de memorias micro-SD y un conector de pines para expansión en una configuración $2 \times 10$. El conector fue diseñado para interconectar al microcontrolador con los sensores, el GPS, LoRa y FPGA. En este conector se tienen disponibles los protocolos de comunicación serial: I2C, Serial Asíncrono y SPI. El puerto I2C se utiliza para el intercambio de datos con los sensores de presión, humedad, temperatura, acelerómetro, giroscopio y magnetómetro.

El puerto serial asíncrono se utiliza para la comunicación con el GPS. El puerto SPI se emplea para la comunicación de datos con el LoRa y con el FPGA. Adicionalmente, el conector permite el control de un LED-RGB incorporado en el módulo de sensores. Además dispone de pines de control, para activar o desactivar los módulos GPS y FPGA, y posee pines de propósito general para una posible adición de hardware de expansión.

Adicional a este conector, el módulo cuenta con dos conectores micro-USB estándar destinados para el usuario programador. Un conector corresponde a un puerto de comunicación de clase USB-CDC. El otro conector corresponde a un puerto USB-OTG que es utilizado para interactuar con el sistema operativo del microcontrolador. La distribución de los componentes del módulo se pueden observar en la Figura 3. En el mismo módulo se integra una fuente conmutada de $1 \mathrm{~A}$ de capacidad de corriente, que suministra un voltaje de $3.3 \mathrm{~V}$ hacia el microcontrolador y hacia todos los demás módulos del CanSat, a través del conector de expansión.

El interior del microcontrolador está constituido por un procesador ARM de 32 bits de la familia Cortex-M4 y una arquitectura de periféricos constituida por: 14 timers de propósito general, 3 puertos seriales asíncronos, 3 puertos SPI, 3 puertos I2C, 88 puertos GPIO, entre otros. El procesador es impulsado por un cristal de $8 \mathrm{MHz}$ de frecuencia, que internamente puede ser configurada a $168 \mathrm{MHz}$. Este microcontrolador cuenta con una unidad de procesamiento de punto flotante (FPU), que permite realizar cálculos aritméticos de 32 bits por hardware. 


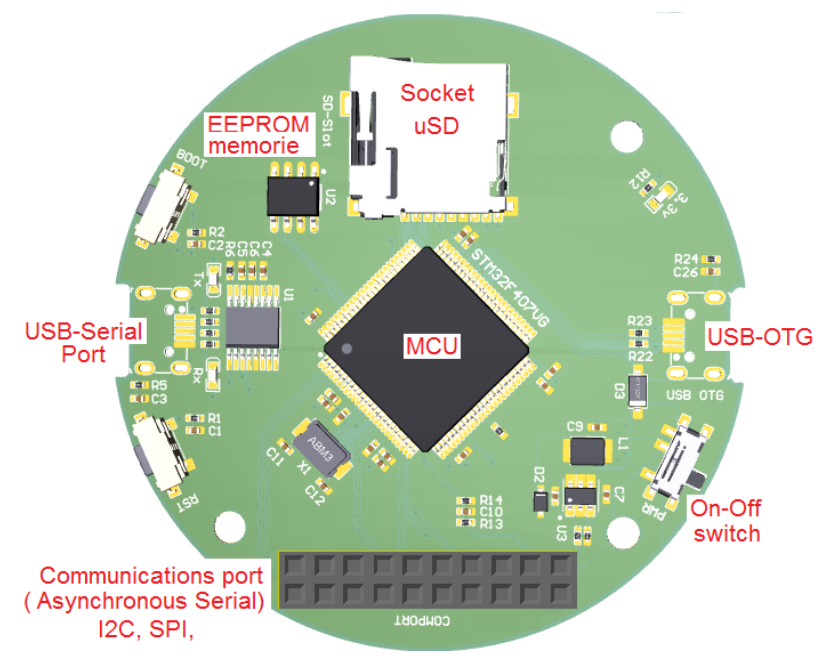

Figura 3 Distribución de componentes del módulo de control

Fuente: Elaboración Propia

Como sistema operativo se utiliza el intérprete Micropython (Damien P. George, 2019). Este sistema está diseñado para ejecutarse en sistemas embebidos basados en microcontroladores. Mediante este intérprete se pueden ejecutar códigos de programa en lenguaje Python V3, lo que le confiere un sistema amigable y potente para la construcción de programas para el control del CanSat. Micropython permite la programación de código en tiempo de ejecución o mediante scripts.

En general la unidad de control, se encarga de gestionar los tiempos de muestreo de cada sensor. También gestiona el preprocesamiento de los datos y los conforma en una trama de datos, para su transmisión hacia la estación terrena. Se emplea una tasa de 10 muestras por segundo. $\mathrm{Y}$ en la trama se transmiten los datos de: aceleración de 3 ejes, velocidad angular de 3 ejes, orientación magnética, temperatura, presión atmosférica, humedad relativa, altitud, longitud, latitud, velocidad, nivel de batería y el tiempo universal coordinado.

Los datos son ensamblados en una trama con una longitud máxima de 255 caracteres en ASCII. Cada dato es separado mediante un identificador inicial compuesto por dos palabras mayúsculas y un carácter coma. Los identificadores que se emplean para el envío de datos se muestran en la Tabla 1. La trama es cargada al buffer de transmisión del dispositivo de comunicaciones, para su transmisión bajo el estándar de comunicación LoRa ${ }^{\circledR}$ (Semtech, 2019).
El programa principal de control, así como las librerías de control de los sensores, son cargados a una memoria micro SD de 4 GB de capacidad, en formato FAT32. Esta memoria es insertada al socket del módulo de control. Desde la memoria el microcontrolador ejecuta el script principal llamado "main.py". En este archivo se invocan las librerías de control de cada sensor y/o módulo. Además se gestiona el momento de transmisión de datos hacia la estación terrena, así mismo, controla el tiempo en que se manda la orden para la captura de fotos.

\begin{tabular}{|l|l|}
\hline \multicolumn{2}{|c|}{ Identificador } \\
\hline AX, AY, AZ & $\begin{array}{l}\text { Aceleración en los 3 ejes } \\
\text { (Acelerómetro). }\end{array}$ \\
\hline GX, GY, GZ & $\begin{array}{l}\text { Velocidad angular en los 3 ejes } \\
\text { (Giroscopio). }\end{array}$ \\
\hline MX, MY, MZ & $\begin{array}{l}\text { Orientación magnética (Brújula } \\
\text { Magnética). }\end{array}$ \\
\hline LA & Latitud Geográfica (GPS). \\
\hline LO & Longitud Geográfica (GPS). \\
\hline AL & Altura (GPS). \\
\hline VE & Velocidad (GPS). \\
\hline TI & Tiempo Universal Coordinado (GPS). \\
\hline T1 & Temperatura externa 1 (TMP102). \\
\hline BT & Temperatura externa 2 (BME280). \\
\hline BH & Humedad Relativa (BME280). \\
\hline BB & Presión Atmosférica (BME280). \\
\hline BA & Voltaje de la batería (MCU) \\
\hline
\end{tabular}

Tabla 1 Identificadores utilizados para la construcción de la trama de datos

Fuente: Elaboración Propia

\section{Módulo de sensores}

Este módulo incorpora todos los sensores de medición de variables físicas y de posicionamiento geográfico, que se utilizan en el CanSat. El módulo se divide en tres secciones principales: sensores inerciales, sensores de clima y sensor de posicionamiento GPS. La distribución de las regiones en la placa PCB se muestra en la Figura 4.

\section{Sensores inerciales}

La placa de los sensores inerciales está conformada por el circuito MPU6050 de InvenSense, y el circuito AK8975 de AsahiKASEI. El primero es un acelerómetro y giroscopio de 3 ejes cada uno, es decir de 6 grados de libertad en total. El acelerómetro permite medir magnitudes de aceleración comprendidas entre rangos de $\pm 2, \pm 4, \pm 8$ y \pm 16 gravedades; mientras que el giroscopio puede medir velocidades angulares en el rango de $\pm 250, \pm 500, \pm 1000 \mathrm{y} \pm 2000^{\circ} / \mathrm{seg}$. 
El MPU6050 posee un puerto I2C principal y uno esclavo, ambos configurados a una velocidad de transferencia de $400 \mathrm{Kbits} / \mathrm{s}$. Mediante el puerto I2C principal, el módulo de control configura los rangos de medición en el sensor y también lee los datos crudos del sensor. El puerto I2C esclavo es utilizado para conectar el circuito AK8975. El AK8975 es un compás electrónico de 3 ejes, que incorpora sensores para detectar el campo magnético terrestre. Este sensor puede medir en un rango de $\pm 1200 \mu \mathrm{T}$. Los datos provenientes del sensor son transferidos hacia el MPU6050 por el puerto I2C esclavo. El acelerómetro es configurado en el rango de medición de $\pm 16 \mathrm{G}$. El giroscopio es configurado para un rango de $\pm 250^{\circ} / \mathrm{seg}$. En ambos sensores la frecuencia de muestreo es fijada en $20 \mathrm{~Hz}$. El compás magnético es configurado para una frecuencia de muestreo de $8 \mathrm{~Hz}$. La posición de ambos circuitos integrados es ubicada para que el eje cartesiano $\mathrm{Z}$, sea perpendicular al plano de la tarjeta $\mathrm{PCB}$, de esta forma, dichos ejes apunten hacia arriba. La orientación de los ejes X e Y se puede observar en la Figura 4.

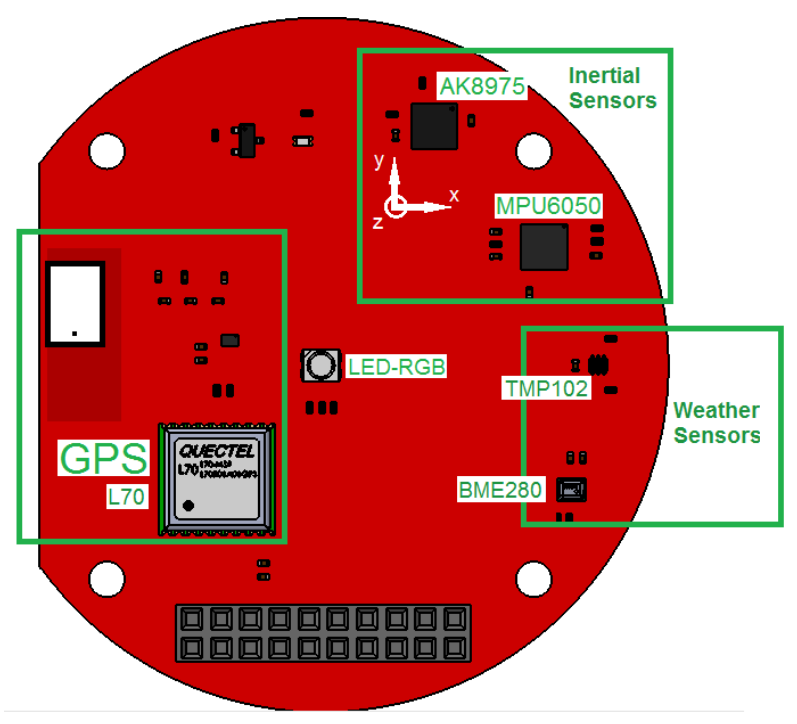

Figura 4 Disposición de componentes del módulo de sensores

Fuente: Elaboración Propia

\section{Sensores de clima}

La placa de sensores que mide el estado del clima la constituyen los circuitos integrados BME280 de BOSCH y el TMP102 de Texas Instrument. El BME280 es un sensor combinado de humedad relativa, presión atmosférica y temperatura ambiente. Sus rangos de medición y error se muestran en la Tabla 2. Este sensor incorpora un puerto I2C mediante el cual se transfieren los datos de medición hacia el módulo de control.

\begin{tabular}{|l|r|r|}
\hline \multicolumn{1}{|c}{ Sensor } & \multicolumn{1}{c|}{$\begin{array}{l}\text { Rango de } \\
\text { medición }\end{array}$} & Exactitud \\
\hline Humedad & 0 a $100 \%$ & $\pm 3 \% \mathrm{RH}$ \\
\hline Temperatura & $-40^{\circ} \mathrm{C} \mathrm{a} 80^{\circ} \mathrm{C}$ & $\pm 1^{\circ} \mathrm{C}$ \\
\hline $\begin{array}{l}\text { Presión } \\
\text { Atmosférica }\end{array}$ & 300 a $1100 \mathrm{hPa}$ & $\pm 1 \mathrm{hPa}$ \\
\hline
\end{tabular}

Tabla 2 Rangos de medición y de error del sensor BME280

Fuente: Elaboración Propia

El sensor proporciona las lecturas de las variables de forma cruda, con una resolución de 20 bits para las variables de humedad y presión atmosférica, y de 16 bits para la temperatura. Estos datos deben ser procesados en la unidad de control, a través de unos parámetros de ajuste, que incorpora el sensor de fábrica. Estos parámetros son leídos del sensor y almacenados, una sola vez, en la memoria RAM del microcontrolador. La frecuencia de muestreo del sensor es fijada en $2 \mathrm{~Hz}$.

El circuito integrado TMP102 es un sensor de temperatura digital. Incorpora una interfaz de comunicación I2C configurado para una tasa de transferencia de $40 \mathrm{kbits} / \mathrm{seg}$. Su resolución es de 12 bits, con un rango de lectura de $-40^{\circ} \mathrm{C}$ hasta $150^{\circ} \mathrm{C}$. Este sensor no requiere de calibración y tiene una exactitud promedio de $\pm 3^{\circ} \mathrm{C}$ sobre todo el rango de medición y de $\pm 2^{\circ} \mathrm{C}$ sobre un rango de $25^{\circ} \mathrm{C}$ a $85^{\circ} \mathrm{C}$.

\section{Sistema de posicionamiento GPS}

El sistema de posicionamiento global o GPS, lo conforma el módulo L70 de Quectel, la antena resonadora y un amplificador de bajo ruido (LNA). La ubicación física de los componentes sobre la placa PCB, se muestra en la Figura 4. Este sistema realiza de forma automática la recepción de las señales satelitales, las procesa y proporciona las variables de posicionamiento.

Tiene la capacidad de recepción de señales del Sistema de Aumentación Basado en Satélites (SBAS), que permiten mejorar el posicionamiento horizontal y vertical del sistema (Van Diggelen, 2009). Se utiliza el puerto serial asíncrono para transmitir los datos hacia el módulo de control (MCU). Este puerto puede configurarse en velocidades de 4800 hasta 115200 baudios y por defecto está configurado a 9600. La tasa de actualización del módulo GPS puede configurarse desde $1 \mathrm{~Hz}$ hasta $10 \mathrm{~Hz}$. 
Este módulo envía los datos de navegación en el protocolo estándar NMEA 0183 (National Marine Electronics Association, s.f.). El protocolo consiste de una trama de caracteres ASCII reconocidos por un identificador de trama. Cada trama envía información diferente y complementaria. Las tramas que se utilizan en el CanSat son las que empiezan con los identificadores GPRMC (Datos de posición mínima recomendada) y GPGGA (Datos fijos del sistema de posicionamiento global).

Ambas tramas envía los datos de Latitud, Longitud y el Tiempo Universal Coordinado (UTC). De manera individual, la trama de GPRMC proporciona también datos de la fecha (día-mes-año), velocidad de traslación (nudos) y orientación sobre la tierra (grados). La trama GPGGA proporciona también datos sobre el número de satélites usados y de la Altura (metros) sobre el Geoide y el Elipsoide de acuerdo al Sistema Geodésico Mundial (WGS84). El MCU del módulo de control extrae de las tramas los datos de Latitud, Longitud, Altura sobre el Geoide, Velocidad y UTC.

\section{Módulo de comunicaciones}

Este módulo está constituido únicamente por un transceptor con tecnología de modulación de espectro extendido, conocida como: LoRa® (Long Range). Esta tecnología es patentada por la empresa SEMTECH, y muchos fabricantes de sistemas de comunicación la han adoptado para ofertar dispositivos de comunicación. El dispositivo LoRa utilizado en este módulo, es el Módulo LoRa RA-02 V.1 del fabricante AiThinker Technology. En la Tabla 3 se presentan las principales características de este dispositivo.

\begin{tabular}{|l|l|}
\hline Especificación & Valor (unidades propias) \\
\hline Puerto de comunicación & SPI \\
\hline Velocidad de transferencia & $\begin{array}{l}\text { Hasta 300 Kbps } \\
\text { (programable) }\end{array}$ \\
\hline $\begin{array}{l}\text { Rango de Frecuencias } \\
\text { (programable) }\end{array}$ & 410 a $525 \mathrm{MHz}$ \\
\hline Ancho de banda & $125 \mathrm{KHz}$ \\
\hline $\begin{array}{l}\text { Potencia máxima de } \\
\text { transmisión }\end{array}$ & $18 \pm 1 \mathrm{dBm}$ \\
\hline $\begin{array}{l}\text { Consumo de corriente } \\
\text { máximo }\end{array}$ & $97 \mathrm{~mA}$ \\
\hline Voltaje de alimentación & 2.5 a 3.7 volts \\
\hline
\end{tabular}

Tabla 3 Especificaciones eléctricas del módulo LoRa RA$02 \mathrm{~V} .1$

Fuente: Elaboración Propia
El dispositivo puede configurarse para transmitir una trama de hasta 256 bytes de longitud. Y realiza un chequeo automático de la integridad de la trama recibida, mediante un código cíclico de redundancia o CRC. También, el dispositivo transmite o recibe en un modo de Hall-Duplex. El MCU es el encargado de gestionar el momento en que el dispositivo se configura en modo transmisor o receptor.

La interface de comunicación entre el LoRa y el MCU es el puerto SPI, que comparte también con el módulo de visión. La velocidad de transferencia de este puerto es fijada en 2 Mbps. Para distinguir entre los dos módulos conectados al bus SPI, el MCU utiliza dos pines GPIO, para seleccionar uno u otro dispositivo, poniendo en un estado lógico bajo el pin del dispositivo de interés. El dispositivo LoRa se controla a través de la escritura/lectura de registros internos. El MCU carga la trama de datos de los sensores, hacia el dispositivo LoRa y configura los registros para que entre en un modo de transmisión. El dispositivo en automático calculará el CRC y transmitirá el paquete. Si por el contrario se desea recibir información, el MCU configura al dispositivo para que "escuche" la recepción de datos. Si un paquete es recibido con éxito, se avisará al MCU mediante el estado lógico del registro de interrupción interno del dispositivo LoRa.

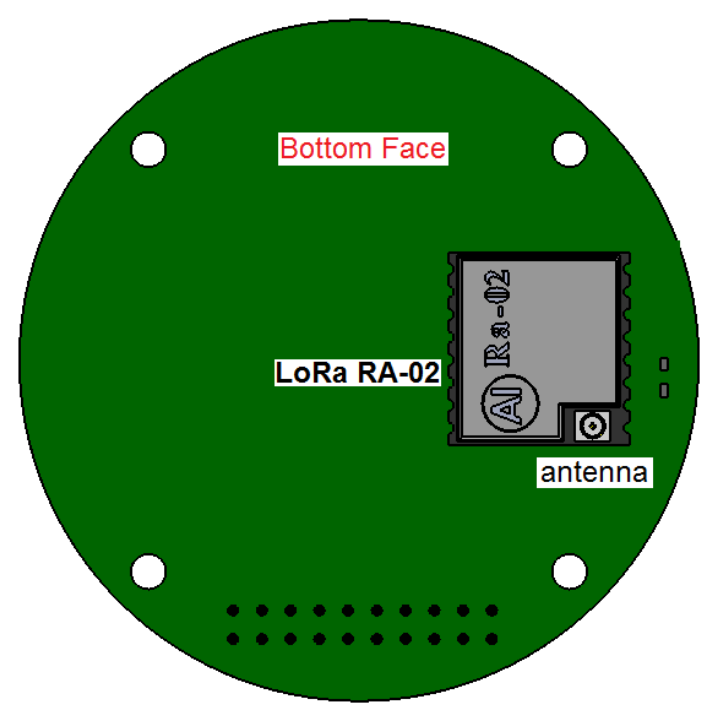

Figura 5 Disposición del módulo LoRa RA-02 Fuente: Elaboración Propia

\section{Modulo de visión}

El módulo de visión está constituido por una cámara OV7670 de Omnivision, interconectada con la FPGA-XC6SLX16 de Xilinx. También se incorporó una memoria SDRAM de $4 \mathrm{Mb}$ por 16 bits, modelo MT48LC4M16.

SÁNCHEZ-TEXIS, Fernando, IBARRA-BONILLA, Mariana Natalia y REYES-CASTILLO, Ivan. Pico satélite educativo CanSat desarrollado en las plataformas ARM-CortexM4 y FPGA. Revista de Aplicaciones de la Ingeniería. 2019. 
Esta memoria se encuentra interconectada directamente al FPGA mediante un bus dedicado de datos y control. El sistema permite guardar hasta 10 fotografías en formato RGB de 24 bits por pixel, con una resolución de 640 x 480 pixeles (VGA). La cámara es igual en resolución y puede transmitir hasta 25 cuadros por segundo. Mediante una IP diseñada para el FPGA, el módulo de visión se comunica a través de un puerto SPI, con el módulo de control. La tasa de transferencia de datos del puerto, está configurada a 2 Mbps.

El módulo acepta comandos de un byte de longitud para indicarle, cuando capturar fotografías (comando: 0x02), transmitirlas (0x03) y/o resetear la memoria SDRAM (0x01). La IP fue desarrollada en lenguaje VHDL e incorpora una máquina de estados dedicada al control de la cámara y al acceso hacia la memoria SDRAM. El FPGA y la memoria SDRAM se encuentran ubicados en la cara superior de la PCB del módulo, mientras que la cámara está ubicada en la cara inferior. En la Figura 6 se muestra un esquema general de este módulo. La cámara es configurada para un enfoque y control de exposición automática, lo que asegura fotografías de la mejor calidad.

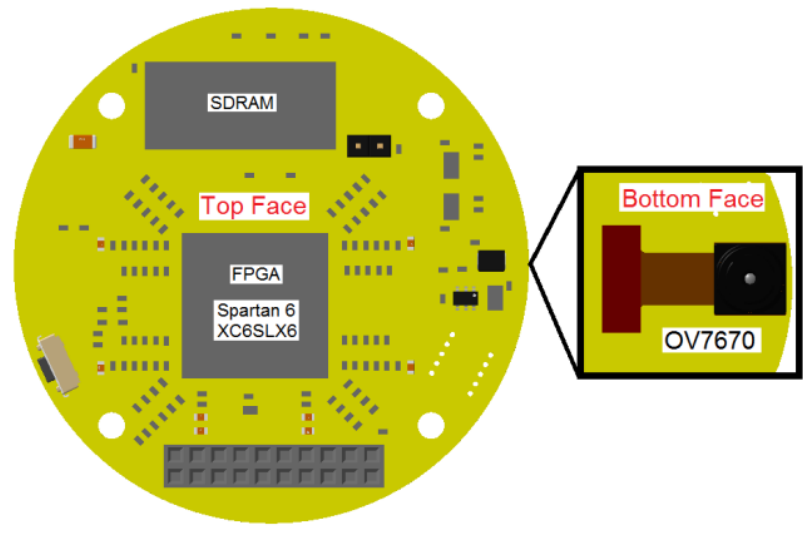

Figura 6 Disposición de componentes del módulo de visión

Fuente: Elaboración Propia

\section{Resultados}

La Figura 7 presenta el resultado de la fabricación y montaje de los componentes para cada módulo que integra el CanSat. En total se construyeron 3 CanSat, los cuales se muestran en la Figura 8. Los módulos de control, comunicación y sensores, se fabricaron en tecnología de circuito impreso de doble capa, con una forma circular de $60 \mathrm{~mm}$ de diámetro y espesor de PCB de $1.6 \mathrm{~mm}$ en un material de fibra de vidrio laminado FR-4.
El módulo de visión fue realizado utilizando la misma tecnología, pero de 4 capas, 2 de ellas para planos de voltaje y tierra y las otras para las vías de señales.
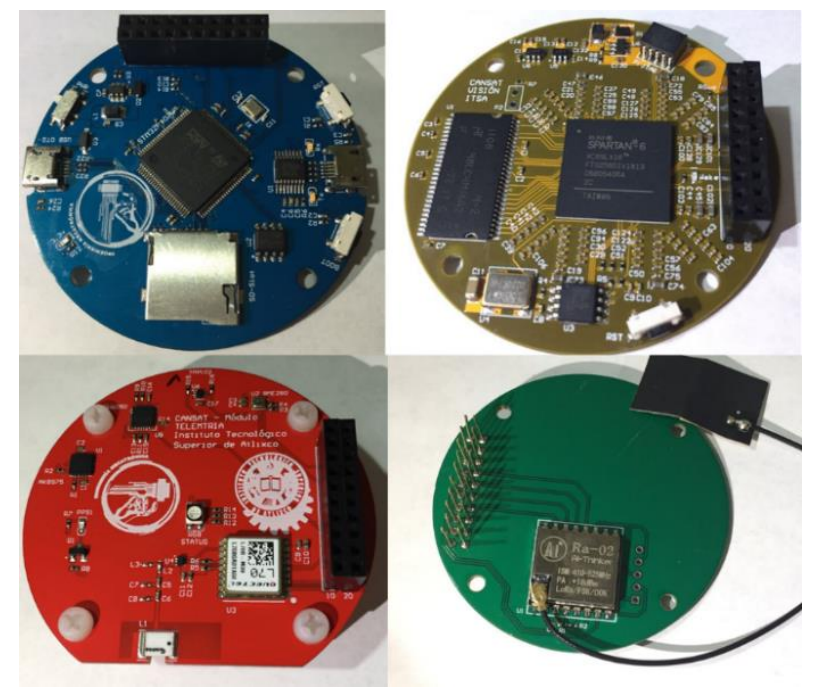

Figura 7 Módulo del CanSat Fuente: Elaboración Propia

Se realizó una prueba de alcance del sistema de comunicaciones. La prueba consistió en posicionar un CanSat en modo receptor, en un edificio a una altura de un piso (aproximadamente $4 \mathrm{~m}$ ). Un segundo CanSat en modo de transmisor se movió en un entorno urbano, entre edificios, arboles, automóviles, interferencias eléctricas, etc. La finalidad de la prueba fue verificar la integridad de los datos, logrando una máxima distancia de $460 \mathrm{~m}$.

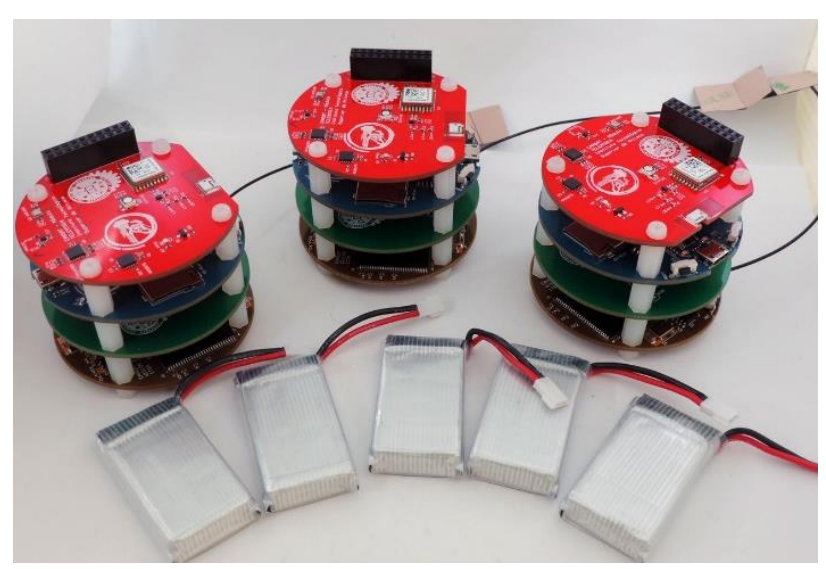

Figura 8 Los CanSat integrados Fuente: Elaboración Propia

La Figura 9 presenta la ruta de la prueba. El estándar LoRa proyecta como distancia mínima de transmisión $5 \mathrm{~km}$ entre dispositivos. Si se compara la distancia real obtenida se observa una pérdida del $90.8 \%$. Esto se debe a las perdidas por absorción de la señal debido a los obstáculos. 
Por ejemplo las estructuras metálicas (automóviles, postes de luminaria, señalización urbana, etc.) afectan considerablemente la potencia irradiada por la antena. Debido a que en los concursos de CanSat, estos se elevan hacia la atmosfera no hay obstáculos que afecten a la señal de transmisión. Por lo que se espera que en una prueba de este tipo, el alcance de la transmisión sea lo que establece el estándar.

Con respecto al módulo de sensores, se comprobó la lectura de todos los parámetros definidos por los indicadores de la Tabla 1. La Figura 10 presenta los datos leídos de los sensores en una consola de comunicación serial que permite ejecutar el lenguaje de Python, por lo que se evidenció el funcionamiento correcto de cada uno de los sensores.

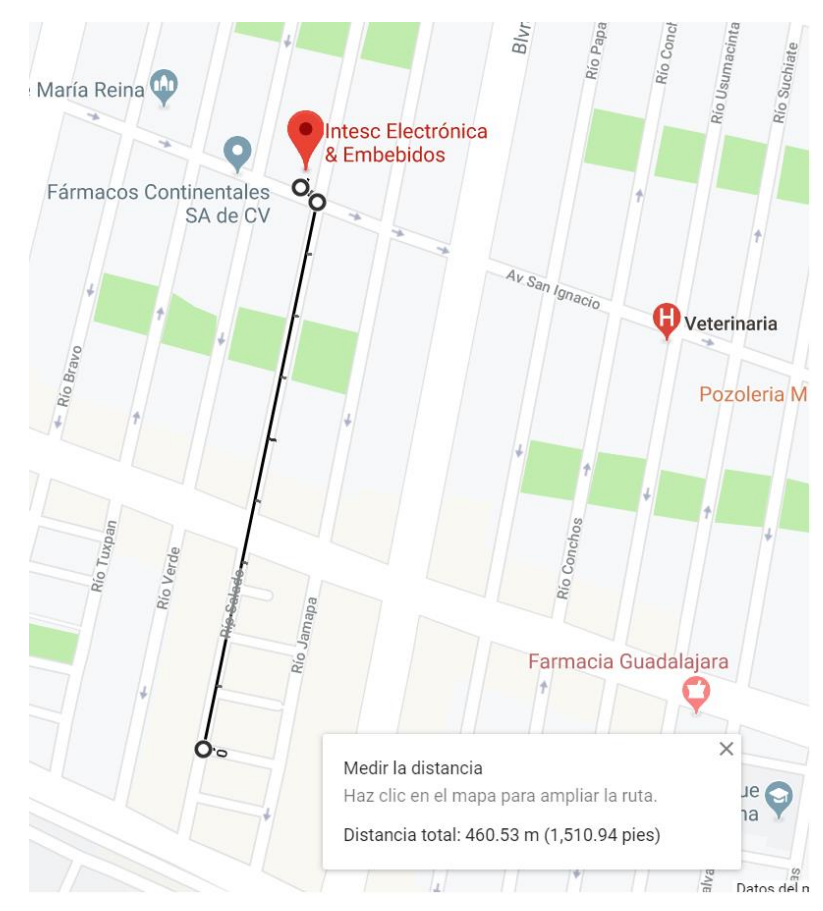

Figura 9 Ruta de la prueba de alcance Fuente: Elaboración Propia

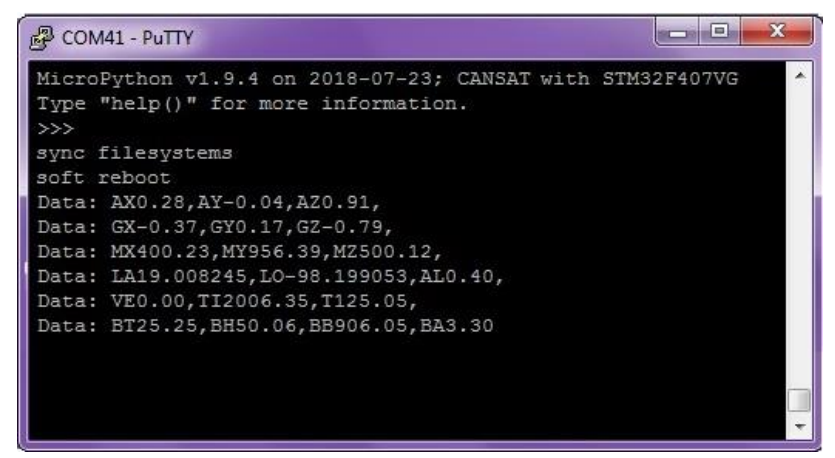

Figura 10 Respuesta del CanSat con los parámetros de los sensores

Fuente: Elaboración Propia

\section{Agradecimiento}

Los autores agradecen el apoyo otorgado por parte de PRODEP, para la realización de este trabajo, el cual fue financiado con el proyecto: 5011-6/17-8773.

\section{Conclusiones}

Se presentó el diseño e implementación de un pico-satélite tipo CanSat en las plataformas ARM-CortexM4 y FPGA Spartan6. La estructura del CanSat consiste de cuatro módulos: control, sensores, comunicación y visión. Todos los módulos se fabricaron en forma circular de $60 \mathrm{~mm}$ y espesor de $1.6 \mathrm{~mm}$ usando tecnología de circuito impreso de montaje superficial, a 2 capas para control, sensores y comunicación, y a 4 capas para visión. El módulo de control incorpora un microcontrolador ARM-STM32F407 integrado con el OS-Micropython que, mediante la programación en lenguaje Python, permite ejecutar la comunicación correcta con los sensores y el sistema de visión con FPGA, y la transmisión de datos por el estándar LoRa. Se ejecutaron pruebas preliminares que nos permiten validar el diseño.

\section{Trabajo futuro}

El trabajo futuro consiste en el desarrollo de la estación terrestre y en la ejecución de las pruebas cuando el CanSat es elevado por un dron. Esto con la finalidad de presentar el CanSat en las competencias.

\section{Referencias}

Bouwmeester, J. y Guo, J. (2010). Survey of worldwide pico-and nanosatellite missions, distributions and subsystem technology. Acta Astronautica, 67(7-8), 854-862.

Carrasco, R. C., y Vázquez, S. V (2014). Nanosatélite basado en microcontroladores PIC: CanSat.

Chaudhry, V. y Mishra, I. (2015). ZENITH: A Nano-Satellite for Atmospheric Monitoring (No. 2015-01-2395). SAE Technical Paper.

Colin, A. y Jimenez-Lizárraga, M. (2015). The CanSat technology for climate Monitoring in small regions at altitudes below $1 \mathrm{~km}$. IAA Climate Change \& Disaster Management Confer ence.

SÁNCHEZ-TEXIS, Fernando, IBARRA-BONILLA, Mariana Natalia y REYES-CASTILLO, Ivan. Pico satélite educativo CanSat desarrollado en las plataformas ARM-CortexM4 y FPGA. Revista de Aplicaciones de la Ingeniería. 2019. 
Damien P.G., P.S. (30 de Junio de 2019). MicroPython documentation. Obtenido de http://docs.micropython.org/en/latest/

National Marine Electronics Association. (s.f.). Recuperado el 01 de Julio de 2019, de https://www.nmea.org/

Paudel, S., Prajapati, R. C., Thapa, J., Shrestha, S., Dutta, A. K., \& Humagain, S. (2017). Development of CanSat Ground-Station using LabVIEW. Proceeding of MARS Summit, India.

Semtech (01 de Julio de 2019). What is LoRa®?. Obtenido de

https://www.semtech.com/lora/what-is-lora

Twiggs, R. (1998). University Space System Symposium (USSS). Hawaii, USA.

Van Diggelen, F. S. T. (2009). A-gps: Assisted gps, gnss, and sbas. Artech House.

Waleligne, M. (2018). Design and Development of CANSAT: Transmit Weather Data from Troposphere Level to The Ground Station (Doctoral dissertation, Addis Ababa University). 